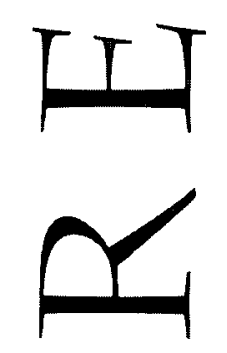

\title{
Pharmaceutical Care: Needed Now More than Ever
}

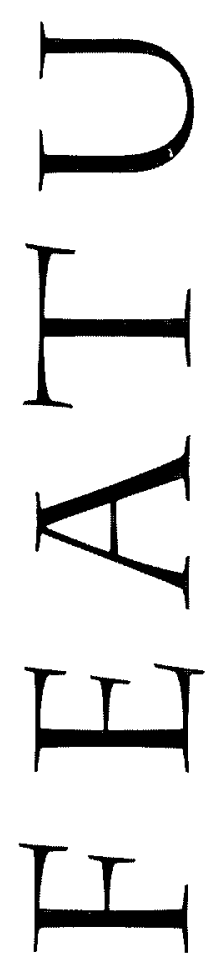

MARY JANE GORE

MARY JANE GORE is a freelance writer based in Charlotiesville, Virginia.

Copyright (O 1995 Academy of Managed Care Pharmacy, Inc. All rights reserved.
$\mathrm{P}$ harmaceutical care-pharmacy's current holy grail-presents special opportunities and challenges to managed care pharmacy. While medical records are readily available in staffmodel HMOs, pharmacists in IPA practices must check carefully for the diseases being treated with pharmacotherapy. The pharmacist in mail-service operations finds written drug information plentiful for dispensing with the product, but the lack of face-to-face contact must be overcome.

But in many ways, managed care presents pharmacists with the opportunity to actualize the principles of pharmaceutical care. In fact, employers and self-insured payers are demanding that they do so--no longer is the ultimate focus on the drug product and the associated dispensing fee, but employers are increasingly concerned about reduced productivity and lost work days when considering the cost of therapy.

"Managed care in principle looks at all of the inputs to the medical care 
process without judging each on its respective price," says Gene Reeder, Ph.D., of the University of South Carolina, who takes a pharmacoeconomic view of the process of pharmaceutical care. "In some cases, you might use an expensive pharmaceutical because that may be the most efficient way of treating the patient and keeping him out of the physician's office or the hospital."

The principles of pharmaceutical care have been applied in many different ways in managed care settings. Let's look at some of these.

\section{CARE WITHIN HMOS AND PPOS -}

Reeder notes that, while a staffmodel HMO can provide access to patient data more quickly than some other managed care arrangements, "they still don't have ready access, because they were organized to manage care, not to manage data or budgets." As the managed care revolution occurs, a variety of forms in delivery systems and economic incentives will emerge, "because no one knows which will be best or if one is the best," says Reeder, professor and director of the university's Center for Pharmacoeconomic Studies.

Bob Rase, Pharm.D., clinical pharmacy coordinator of Humana Health Care Plans of Kansas City, works with several different managed care systems in his region, most of which are staffmodel HMOs, mixed with some IPAs and network HMO models.

"A lot of the requirements for pharmaceutical care are met in a staff model, whereas some are not yet met in the retail network," he says. "We're trying to optimize the overall drug-use process."

As Rase sees it, five discrete steps are involved in the process:

$\boldsymbol{\nabla}$ Deciding to treat, usually done by a physician

$\boldsymbol{\nabla}$ Selecting the drug therapy, an area where pharmacists can have input; $\nabla$ Drug prescribing and dispensing $\boldsymbol{\nabla}$ Taking the drug

$\nabla$ Documenting patient outcomes Training and motivation of pharmacists is essential, and this is especially true among network-model HMOs that use community pharmacies, Rase says. "If your company wants consistently to apply pharmaceutical care, then it must ensure that every pharmacist in the network is able and willing to deliver good pharmaceutical care," he explains. "That is a tall order."

Gary Wirth, assistant director for managed care programs of Giant Discount Drug stores, an East Coast chain, says that these pharmacies have contracts with many different managed care organizations (MCOs). The major challenge is finding an MCO providing the needed integrated medical data feedback to the pharmacy. Wirth hopes to put together a program with a local provider such as an HMO and see which interventions work for benefit of the patient, provider, and pharmacy.

"There is a lot of rhetoric out there, but not a lot of people willing to put forth the effort," he says. "We are very interested in finding a way to make pharmaceutical care work in our stores and with managed care partners."

One way to accomplish this is through data gathered from valid clinical trials, which Giant hopes to run with assistance from the University of Maryland School of Pharmacy, where Giant pharmacists are undergoing mandatory training in pharmaceutical care. "One of our other real challenges in our busy stores is to create the environment in which pharmacists feel capable of giving such care. We have to provide the knowledge and the opportunity," Wirth says.

Diversified Pharmaceutical Services (DPS), a pharmacy benefit management (PBM) company in Bloomington,

Minn., has been able to design interventions best provided through a community pharmacy provider, located either within the DPS national network or a client-managed pharmacy.

"Certain detection parameters facilitate intervention and communicate back to the pharmacist when there is an issue such as potential therapeutic modification, extra counseling, education, or compliance management that should be accomplished through the pharmacist, says DPS's Cindy Anderson, director of pharmaceutical care services. "It taps into the existing process through technol- ogy. The pharmacist wants to be recognized for providing services beyond dispensing, and this can facilitate an incremental approach to more comprehensive pharmaceutical care services."

\section{INFORMATION MANAGEMENT: THE LATEST KEY TO CARE}

Reeder reports a spurt in the number of regional pilot projects on information integration. The Community Health Information Network (CHIN) is an example of several healthcare organizations attempting to share clinical information over a network.

"Everyone is realizing that the key to making all of this work is access to the right kinds of information for decision making and being able to analyze a system so that adjustments and incentives can begin to improve efficiency while maintaining the quality of care," Reeder says.

Rase believes that one of the most basic requirements on the way to total pharmaceutical care is the information infrastructure.

"In a staff model, our clinic is typically on-line with an information system so that the pharmacy can log into all of the information. Or we're in the same building with the medical chart or the physician," he says. "It is about proximity with information and with the people involved. Sometimes the pharmacist in a drugstore is, unfortunately, out of the loop."

\section{THE MAGIC OF PLASTIC}

Patients who carry prescription service cards, such as those issued by PCS of Scottsdale, Ariz., provide a wealth of information to a pharmacist. Claims adjudication, drug-use review (DUR), retrospective or metropolitan statistical area DUR, formulary and rebate programs, disease-management programs, and managed infusion programs are available. In the future, the cards can be developed to contain medical and medication histories of patients.

Pharmacists today are able to transmit prescription claim information, says a PCS representative. The client might have concurrent DUR, formulary, re- 
bate, and exclusion such as a prior authorization program. When the pharmacist enters patient i.d. and carrier group numbers, he or she receives the appropriate messages for DUR, prior authorization, and other aspects of care.

\section{MAIL-SERVICE DIVISIONS DELIVER CARE}

Mail-service divisions have been able to manage several drug-related problems targeted by Hepler and Strand in their definition of pharmaceutical care. Problems such underuse, overuse, and excess dosage amount can be facilitated through a mail-service setting with a pharmacist contacting a physician or a patient about identified problems. Most problems are identified through computerization and patient profile information.

Some measures that address potential drug-related problems can be accomplished through mail-service pharmacy, but other issues cannot be identified through computerization alone. "As pharmaceutical care services evolve, there will be a need for pharmacists to interact directly through the physician and patient about problems to identify drug-related problems that cannot be identified through on-line claims processing," one pharmacist told JMCP.

\section{DISEASE STATE MANAGEMENT -}

Efficient disease management is also a critical aspect of care that presents a new role for pharmacists, as well as new information applications. Whereas pharmacists traditionally had a "transaction" role-filling prescriptions quickly and accurately-now they are accepting responsibility for problem patients who consume inordinate quantities of managed care resources.

MCOs are identifying patients likely to benefit from good pharmacy services, and several are evaluating these interventions to learn to what extent pharmacists have impact on the total utilization and cost. Target conditions include asthma and chronic obstructive lung disease, diabetes, depression, coronary artery disease, and arthritis.

"That's where pharmacy is likely to make an impact. With diabetic or asth- matic patients, proper medication use is so critical to keeping those people out of the emergency room and hospital," Reeder says. "You only have to save a few days in the hospital or a few emergency room visits to pay for a lot of pharmaceuticals-and many hours of pharmaceutical care."

PCS has borrowed the concept of a care manager working with patients using home infusion therapy for its disease-management area. These managers take groups of high-risk patients and work closely with them, call them, educate them about their disease, give them some 'homework,' and also work with their physicians. PCS generates physician newsletters explaining different drug therapy protocols for different disease states and recommending formulary alternatives.

DPS also has programs targeting both pharmacists and physicians in the areas of ulcer therapy, asthma, medication noncompliance, as well as diabetes and cardiovascular management. Selfinstructional educational tools for pharmacists are part of the DPS program to ensure quality pharmaceutical care.

Giant stores are developing a training program for all staff pharmacists at a 24-credit hour workshop given through the University of Maryland School of Pharmacy. Several disease states are studied, but "the biggest emphasis is on patient education, in the areas of patient counseling and prevention of medication mismanagement," says Alicia Martinez, manager of pharmacy support services. Patient compliance, a perennial problem area, is emphasized as well.

\section{REIMBURSEMENT AN ISSUE}

Reimbursement is not yet widely available for cognitive services. "Some programs are willing to reimburse the pharmacist, but the reimbursement generally doesn't cover even the cost of the paperwork," says Wirth of Giant.

He says that the answer lies in an electronic environment at the point of sale. "The National Council for Prescription Drug Programs has a communications standard, but the problem is getting people to adopt that standard and then to use it for reimbursement."

Reeder says the problem is that there are no data to demonstrate the value of what pharmacists do beyond dispensing the drug product. "I think that is what people are working on feverishly now-collecting data. If I'm a payer, you are going to have to prove to me your value in the marketplace."

On the network HMO side, reimbursement is a major issue, says Rase. "We do not currently have any reimbursement schedule in place for cognitive services, but we are looking at some pilot programs in specific therapeutic areas where we would arrive at a reimbursement fee based on a set of guidelines or counseling requirements that are fulfilled."

Both pharmacies and MCOs operate with lean budgets. Wirth says that MCOs do not have the staff to support the studies or gather the data through their systems about such variables as primary and secondary diagnoses, medical costs over a period of time, and relevant pharmacy interventions. "They have their own workload and it is hard to get people to work on something like this when there is not an immediate, obvious benefit."

\section{ON THE RIGHT TRACK}

Rase stresses that pharmaceutical care overall doesn't have to be complicated. "Only a lew things must be in place-the right information system available to the right people, who are trained and prepared; and the documentation and reimbursement mechanism in place for pharmacy." Speaking for many, he says, "Down the road, we hope pharmacist activities yield positive outcomes data. We're getting there."

Reeder says that money is a real issue. "Fee for service is a thing of the past. That is one of the inefficiencies, because the incentive is to provide more service. You don't stay in business by providing more care or the wrong care." Tailored care at low costs is key; Reeder notes, the current climate encouraging pharmaceutical care "is a golden opportunity for pharmacists to do what they are uniquely trained to do." 